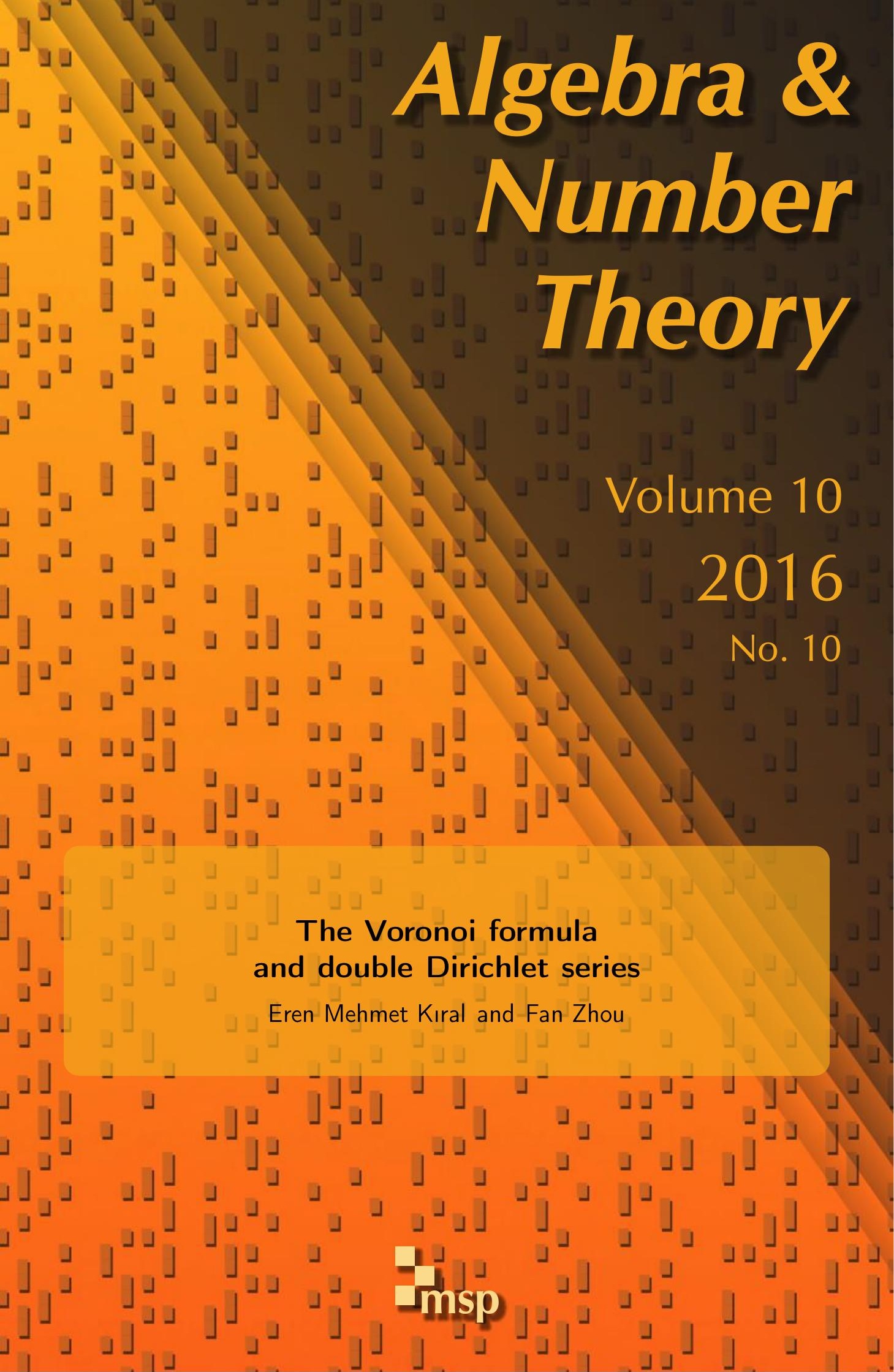




\title{
The Voronoi formula and double Dirichlet series
}

\author{
Eren Mehmet Kıral and Fan Zhou
}

\begin{abstract}
We prove a Voronoi formula for coefficients of a large class of $L$-functions including Maass cusp forms, Rankin-Selberg convolutions, and certain noncuspidal forms. Our proof is based on the functional equations of $L$-functions twisted by Dirichlet characters and does not directly depend on automorphy. Hence it has wider application than previous proofs. The key ingredient is the construction of a double Dirichlet series.
\end{abstract}

\section{Introduction}

A Voronoi formula is an identity involving Fourier coefficients of automorphic forms, with the coefficients twisted by additive characters on either side. A history of the Voronoi formula can be found in [Miller and Schmid 2004]. Since its introduction in [loc. cit.], the Voronoi formula on GL(3) of Miller and Schmid has become a standard tool in the study of $L$-functions arising from GL(3), and has found important applications such as those in [Blomer 2012; Blomer et al. 2013; Khan 2012; Li 2009; 2011; Li and Young 2012; Miller 2006; Munshi 2013; 2015]. As of yet the general GL $(N)$ formula has had fewer applications, a notable one being found in [Kowalski and Ricotta 2014].

The first proof of a Voronoi formula on GL(3) was found by Miller and Schmid [2006] using the theory of automorphic distributions. Later, a Voronoi formula was established for $\operatorname{GL}(N)$ with $N \geq 4$ in [Goldfeld and Li 2006; 2008; Miller and Schmid 2011], with [Miller and Schmid 2011] being more general and earlier than [Goldfeld and Li 2008] (see the addendum there). Goldfeld and Li's proof [2008] is more akin to the classical proof in GL(2) [Good 1981], obtaining the associated Dirichlet series through a shifted "vertical" period integral and making use of automorphy. An adelic version was established by Ichino and Templier [2013], allowing ramifications and applications to number fields. Another direction

MSC2010: primary 11F30; secondary 11F68, 11L05.

Keywords: Voronoi formula, automorphic form, Maass form, multiple Dirichlet series, Gauss sum,

Kloosterman sum, Rankin-Selberg $L$-function. 
of generalization with more complicated additive twists on either side has been considered in an unpublished work of Li and Miller and in [Zhou 2016].

In this article, we prove a Voronoi formula for a large class of automorphic objects or $L$-functions, including cusp forms for $\operatorname{SL}(N, \mathbb{Z})$, Rankin-Selberg convolutions, and certain noncuspidal forms. Previous works [Miller and Schmid 2011; Goldfeld and Li 2008; Ichino and Templier 2013] do not offer a Voronoi formula for RankinSelberg convolutions or noncuspidal forms. Even for Maass cusp forms, our new proof is shorter than any previous one, and uses a completely different set of techniques.

Let us briefly summarize our method of proof. We first reduce the statement of a Voronoi formula to a formula involving Gauss sums of Dirichlet characters. We construct a complex function of two variables and write it as double Dirichlet series in two different ways by applying a functional equation. Using the uniqueness theorem of Dirichlet series, we get an identity between coefficients of these two double Dirichlet series. This leads us to the Voronoi formula with Gauss sums.

One of our key steps in obtaining the Voronoi formula is the use of functional equations of $L$-functions twisted by Dirichlet characters. The relationship between the Dirichlet twists and the additive twists was expected, but not fully understood, such as in [Duke and Iwaniec 1990; Goldfeld and Li 2006, Section 4; Buttcane and Khan 2015; Zhou 2016]. In these works, only prime modulus is dealt with, which is a significant restriction. Miller and Schmid [2006, Section 6] derived the functional equation of $L$-functions twisted by a Dirichlet character of prime conductor from the Voronoi formula. However there is a combinatorial difficulty in reversing this process, i.e., obtaining additive twists of general nonprime conductors from multiplicative ones, which was acknowledged in both [Miller and Schmid 2006, p. 430] and [Ichino and Templier 2013, p. 68]. The method presented here is able to overcome this difficulty by discovering an interlocking structure among a family of Voronoi formulas with different conductors.

Our proof of the Voronoi formula is complete for additive twists of all conductors, prime or not, and unlike [Ichino and Templier 2013], [Miller and Schmid 2006], or [Miller and Schmid 2011], does not depend directly on automorphy of the cusp forms. This fact allows us to apply our theorem to many conjectural Langlands functorial transfers. For example, the Rankin-Selberg convolutions (also called functorial products) for $\mathrm{GL}(m) \times \mathrm{GL}(n)$ are not yet known to be automorphic on $\mathrm{GL}(m \times n)$ in general. Yet we know the functional equations of $\mathrm{GL}(m) \times \mathrm{GL}(n)$ $L$-functions twisted by Dirichlet characters. Thus, our proof provides a Voronoi formula for the Rankin-Selberg convolutions on GL $(m) \times \mathrm{GL}(n)$ (see Example 1.7). Voronoi formulas for these functorial cases are unavailable from [Goldfeld and $\mathrm{Li}$ 2008], [Miller and Schmid 2011] or [Ichino and Templier 2013]. In Theorem 1.3 we reformulate our Voronoi formula like the classical converse theorem of Weil, i.e., 
assuming every $L$-function twisted by a Dirichlet character is entire, has an Euler product (or satisfies Hecke relations), and satisfies the precise functional equations, then the Voronoi formula as in Theorem 1.1 is valid. We do not have to assume it is a standard $L$-function coming from a cusp form.

Furthermore, by Theorem 1.3, we obtain a Voronoi formula for certain noncuspidal forms, such as isobaric sums (see Example 1.8). This is not readily available from any previous work but it is believed (see [Miller and Schmid 2011, p. 176]) that one may derive a formula by using formulas on smaller groups through a possibly complicated procedure. Such complication does not occur in our method because we work directly with $L$-functions.

We first state the main results for Maass cusp forms. Denote

$$
e(x):=\exp (2 \pi i x)
$$

for $x \in \mathbb{R}$. Let $N \geq 3$ be an integer. Let $a, n \in \mathbb{Z}, c \in \mathbb{N}$ and let

$$
\boldsymbol{q}=\left(q_{1}, q_{2}, \ldots, q_{N-2}\right) \quad \text { and } \quad \boldsymbol{d}=\left(d_{1}, d_{2}, \ldots, d_{N-2}\right)
$$

be tuples of positive integers satisfying the divisibility conditions

$$
d_{1}\left|q_{1} c, \quad d_{2}\right| \frac{q_{1} q_{2} c}{d_{1}}, \quad \ldots, \quad d_{N-2} \mid \frac{q_{1} \cdots q_{N-2} c}{d_{1} \cdots d_{N-3}} .
$$

In this case, to simplify notation we set

$$
\xi_{i}:=\frac{q_{1} \cdots q_{i} c}{d_{1} \cdots d_{i}}
$$

Define the hyper-Kloosterman sum as

$$
\begin{aligned}
\mathrm{Kl}(a, n, c ; \boldsymbol{q}, \boldsymbol{d})=\sum_{x_{1} \bmod \xi_{1}}^{*} \sum_{x_{2} \bmod \xi_{2}}^{*} \ldots \sum_{x_{N-2} \bmod \xi_{N-2}}^{*} \\
\quad e\left(\frac{d_{1} x_{1} a}{c}+\frac{d_{2} x_{2} \overline{x_{1}}}{\xi_{1}}+\cdots+\frac{d_{N-2} x_{N-2} \overline{x_{N-3}}}{\xi_{N-3}}+\frac{n \overline{x_{N-2}}}{\xi_{N-2}}\right),
\end{aligned}
$$

where $\sum^{*}$ indicates that the summation is over reduced residue classes, and $\overline{x_{i}}$ denotes the multiplicative inverse of $x_{i}$ modulo $\xi_{i}$. When $N=3, \operatorname{Kl}\left(a, n, c ; q_{1}, d_{1}\right)$ becomes the classical Kloosterman sum $S\left(a q_{1}, n ; \xi_{1}\right)$. For the degenerate case $N=2$, we define $\operatorname{Kl}(a, n, c ;):,=e(a n / c)$.

Let $F$ be a Hecke-Maass cusp form for $\operatorname{SL}(N, \mathbb{Z})$ with the spectral parameters $\left(\lambda_{1}, \ldots, \lambda_{N}\right) \in \mathbb{C}^{N}$. Let $A\left(m_{1}, \ldots, m_{N-1}\right)$, with $\left(m_{1}, \ldots, m_{N-1}\right) \in \mathbb{N}^{N-1}$, be the Fourier-Whittaker coefficients of $F$ normalized as $A(1, \ldots, 1)=1$. We refer to [Goldfeld 2006] for the definitions and the basic results of Maass forms for $\operatorname{SL}(N, \mathbb{Z})$. 
The Fourier coefficients satisfy the Hecke relations

$$
A\left(m_{1} m_{1}^{\prime}, \ldots, m_{N-1} m_{N-1}^{\prime}\right)=A\left(m_{1}, \ldots, m_{N-1}\right) A\left(m_{1}^{\prime}, \ldots, m_{N-1}^{\prime}\right)
$$

if $\left(m_{1} \cdots m_{N-1}, m_{1}^{\prime} \cdots m_{N-1}^{\prime}\right)=1$ is satisfied, $A(1, \ldots, 1, n) A\left(m_{N-1}, \ldots, m_{1}\right)$

$$
=\sum_{\substack{d_{0} \ldots d_{N-1}=n \\ d_{1}\left|m_{1}, \ldots, d_{N-1}\right| m_{N-1}}} A\left(\frac{m_{N-1} d_{N-2}}{d_{N-1}}, \ldots, \frac{m_{2} d_{1}}{d_{2}}, \frac{m_{1} d_{0}}{d_{1}}\right),
$$

and

$$
\begin{gathered}
A(n, 1, \ldots, 1) A\left(m_{1}, \ldots, m_{N-1}\right) \\
=\sum_{\substack{d_{0} \cdots d_{N-1}=n \\
d_{1}\left|m_{1}, \ldots, d_{N-1}\right| m_{N-1}}} A\left(\frac{m_{1} d_{0}}{d_{1}}, \frac{m_{2} d_{1}}{d_{2}}, \ldots, \frac{m_{N-1} d_{N-2}}{d_{N-1}}\right) .
\end{gathered}
$$

The dual Maass form of $F$ is denoted by $\widetilde{F}$. Let $B(*, \ldots, *)$ be the FourierWhittaker coefficients of $\widetilde{F}$. These coefficients satisfy

$$
B\left(m_{1}, \ldots, m_{N-1}\right)=A\left(m_{N-1}, \ldots, m_{1}\right) .
$$

Define the ratio of Gamma factors

$$
G_{ \pm}(s):=i^{-N \delta} \pi^{-N(1 / 2-s)} \prod_{j=1}^{N} \Gamma\left(\frac{\delta+1-s-\overline{\lambda_{j}}}{2}\right) \Gamma\left(\frac{\delta+s-\lambda_{j}}{2}\right)^{-1}
$$

where for even Maass forms, we define $\delta=0$ in $G_{+}$and $\delta=1$ in $G_{-}$, and for odd Maass forms, we define $\delta=1$ in $G_{+}$and $\delta=0$ in $G_{-}$. We refer to [Goldfeld 2006, Section 9.2] for the definition of even and odd Maass forms.

Theorem 1.1 (Voronoi formula on GL $(N)$ of Miller and Schmid [2011]). Let $F$ be a Hecke-Maass cusp form with coefficients $A(*, \ldots, *)$, and $G_{ \pm}$a ratio of Gamma factors as in (6). Let $c>0$ be an integer and let a be any integer with $(a, c)=1$. Denote by $\bar{a}$ the multiplicative inverse of a modulo $c$. Let the additively twisted Dirichlet series be given as

$$
L_{q}\left(s, F, \frac{a}{c}\right)=\sum_{n=1}^{\infty} \frac{A\left(q_{N-2}, \ldots, q_{1}, n\right)}{n^{s}} e\left(\frac{\bar{a} n}{c}\right)
$$

for $\Re(s)>1$. This Dirichlet series has an analytic continuation to all $s \in \mathbb{C}$ and satisfies the functional equation 


$$
\begin{aligned}
& L_{\boldsymbol{q}}(s, F, a / c) \\
& =\frac{G_{+}(s)-G_{-}(s)}{2} \sum_{d_{1} \mid q_{1} c} \sum_{d_{2} \mid \frac{q_{1} q_{2} c}{d_{1}}} \ldots \sum_{d_{N-2} \mid \frac{q_{1} \cdots q_{N-2}}{d_{1} \cdots d_{N-3}}} \\
& \quad \sum_{n=1}^{\infty} \frac{A\left(n, d_{N-2}, \ldots, d_{2}, d_{1}\right) \mathrm{Kl}(a, n, c ; \boldsymbol{q}, \boldsymbol{d})}{n^{1-s} c^{N s-1} d_{1} d_{2} \cdots d_{N-2}} \frac{d_{1}^{(N-1) s} d_{2}^{(N-2) s} \cdots d_{N-2}^{2 s}}{q_{1}^{(N-2) s} q_{2}^{(N-3) s} \cdots q_{N-2}^{s}} \\
& +\frac{G_{+}(s)+G_{-}(s)}{2} \sum_{d_{1} \mid q_{1} c} \sum_{d_{2} \mid \frac{q_{1} q_{2} c}{d_{1}}} \cdots \sum_{d_{N-2} \mid \frac{q_{1} \cdots q_{N-2}}{d_{1} \cdots d_{N-3}}} \\
& \quad \sum_{n=1}^{\infty} \frac{A\left(n, d_{N-2}, \ldots, d_{2}, d_{1}\right) \operatorname{Kl}(a,-n, c ; \boldsymbol{q}, \boldsymbol{d})}{n^{1-s} c^{N s-1} d_{1} d_{2} \cdots d_{N-2}} \frac{d_{1}^{(N-1) s} d_{2}^{(N-2) s} \cdots d_{N-2}^{2 s}}{q_{1}^{(N-2) s} q_{2}^{(N-3) s} \cdots q_{N-2}^{s}}
\end{aligned}
$$

in the region of convergence of the expression on the right-hand side $(\Re(s)<0)$.

The traditional Voronoi formula, involving weight functions instead of Dirichlet series, is obtained after taking an inverse Mellin transform against a suitable test function.

Choose a Dirichlet character $\chi$ modulo $c$, which is not necessarily primitive, multiply both sides of (8) by $\chi(a)$, and sum this equality over the reduced residue system modulo $c$. We obtain the following Voronoi formula with Gauss sums. In Section 3B we show through elementary finite arithmetic that the formulas (8) and (11) are equivalent.

Theorem 1.2 (Voronoi formula with Gauss sums). Let $\chi$ be a Dirichlet character modulo $c$, induced from the primitive character $\chi^{*}$ modulo $c^{*}$ with $c^{*} \mid c$. Define for $\boldsymbol{q}=\left(q_{1}, \ldots, q_{N-2}\right)$ a tuple of positive integers

$$
H\left(\boldsymbol{q} ; c, \chi^{*}, s\right)=\sum_{n=1}^{\infty} \frac{A\left(q_{N-2}, \ldots, q_{1}, n\right) g\left(\overline{\chi^{*}}, c, n\right)}{n^{s}\left(c / c^{*}\right)^{1-2 s}}
$$

for $\mathfrak{R}(s)>1$, and

$$
\begin{gathered}
G\left(\boldsymbol{q} ; c, \chi^{*}, s\right)=\frac{G(s) \chi^{*}(-1)}{c^{N s-1}\left(c / c^{*}\right)^{1-2 s}} \sum_{d_{1} c^{*} \mid q_{1} c} \sum_{d_{2} c^{*} \mid \frac{q_{1} q_{2} c}{d_{1}}} \ldots \sum_{d_{N-2} c^{*} \mid \frac{q_{1} \cdots q_{N-2}}{d_{1} \cdots d_{N-3}}} \\
\sum_{n=1}^{\infty} \frac{A\left(n, d_{N-2}, \ldots, d_{1}\right)}{n^{1-s} d_{1} d_{2} \cdots d_{N-2}} \frac{d_{1}^{(N-1) s} d_{2}^{(N-2) s} \cdots d_{N-2}^{2 s}}{q_{1}^{(N-2) s} q_{2}^{(N-3) s} \cdots q_{N-2}^{s}} \\
\quad \times g\left(\chi^{*}, c, d_{1}\right) g\left(\chi^{*}, \xi_{1}, d_{2}\right) \cdots g\left(\chi^{*}, \xi_{N-3}, d_{N-2}\right) g\left(\chi^{*}, \xi_{N-2}, n\right)
\end{gathered}
$$

for $\Re(s)<0$, where $G$ equals $G_{+}$or $G_{-}$depending on whether $\chi^{*}(-1)$ is 1 or -1 , and $g\left(\chi^{*}, \ell c^{*}, *\right)$ is the Gauss sum of the induced character modulo $\ell c^{*}$ from $\chi^{*}$, 
which is defined in Definition 2.1. Both functions have analytic continuation to all $s \in \mathbb{C}$, and the equality

$$
H\left(\boldsymbol{q} ; c, \chi^{*}, s\right)=G\left(\boldsymbol{q} ; c, \chi^{*}, s\right)
$$

is satisfied.

In proving (11), we define

$$
Z(s, w)=\frac{L_{q}(2 w-s, F) L\left(s, F \times \chi^{*}\right)}{L\left(2 w-2 s+1, \overline{\chi^{*}}\right)},
$$

where $\boldsymbol{q}=\left(q_{1}, \ldots, q_{N-2}\right)$ is a tuple of positive integers, and the function $L_{\boldsymbol{q}}(s, F)$ is given as the Dirichlet series

$$
L_{\boldsymbol{q}}(s, F)=\sum_{n=1}^{\infty} \frac{A\left(q_{N-2}, \ldots, q_{1}, n\right)}{n^{s}}
$$

for $\Re(s) \gg 1$. We express $Z(s, w)$ as a double Dirichlet series in two different ways. In one region of convergence we express the $L$-functions as Dirichlet series and obtain

$$
Z(s, w)=\sum_{n=1}^{\infty} \frac{a_{n}(s)}{n^{2 w}} .
$$

On the other hand, we apply the functional equation of $L\left(s, F \times \chi^{*}\right)$, replacing $s$ with $1-s$, and write $Z(s, w)$ as the Dirichlet series

$$
Z(s, w)=\sum_{n} \frac{b_{n}(s)}{n^{2 w}} .
$$

By the uniqueness of Dirichlet series, we must have $a_{n}(s)=b_{n}(s)$. This equality leads us to the Voronoi formula with Gauss sums.

Our proof only uses the Hecke relations about the Fourier coefficients of $F$ and the exact form of the functional equations. The expression of Gamma factors, or the automorphy of $F$, plays no role. Hence we can formulate our theorem in a style similar to the classical converse theorem of Weil. First, let us list the properties of Fourier coefficients that we use in order to state the following theorem.

The Fourier coefficients of $F$ grow moderately, i.e.,

$$
A\left(m_{1}, \ldots, m_{N-1}\right) \ll\left(m_{1} \cdots m_{N-1}\right)^{\sigma}
$$

for some $\sigma>0$. Given a primitive Dirichlet character $\chi^{*}$ modulo $c^{*}$, define the twisted $L$-function

$$
L\left(s, F \times \chi^{*}\right)=\sum_{n=1}^{\infty} \frac{A(1, \ldots, 1, n) \chi^{*}(n)}{n^{s}}
$$


for $\Re(s)>\sigma+1$. It has analytic continuation to the whole complex plane, and satisfies the functional equation

$$
L\left(s, F \times \chi^{*}\right)=\tau\left(\chi^{*}\right)^{N} c^{*-N s} G(s) L\left(1-s, \widetilde{F} \times \overline{\chi^{*}}\right),
$$

where $G(s)=G_{+}(s)$ or $G_{-}(s)$ depending on whether $\chi^{*}(-1)=1$ or -1 .

Theorem 1.3. Let $F$ be a symbol and assume that with $F$ come numbers

$$
A\left(m_{1}, \ldots, m_{N-1}\right) \in \mathbb{C}
$$

attached to every $(N-1)$-tuple $\left(m_{1}, \ldots, m_{N-1}\right)$ of natural numbers. Assume $A(1, \ldots, 1)=1$.

Assume that these "coefficients" $A(*, \ldots, *)$ satisfy the aforementioned Hecke relations (2), (3) and (4). Further assume that they grow moderately as in (13).

Let $\widetilde{F}$ be another symbol whose associated coefficients $B(*, \ldots, *) \in \mathbb{C}$ are given as in (5) and assume that they also satisfy the same properties. Further, assume that there are two meromorphic functions $G_{+}(s)$ and $G_{-}(s)$ associated to the pair $(F, \widetilde{F})$, so that for a given primitive character $\chi^{*}$, the function $L\left(s, F \times \chi^{*}\right)$ as defined in (14) satisfies the functional equation (15).

Under all these assumptions, $L_{\boldsymbol{q}}(s, F, a / c)$, defined as in (7) for $\Re(s)>1+\sigma$, has analytic continuation to all $s \in \mathbb{C}$, and satisfies the Voronoi formula (8). (The Dirichlet series on the right side of (8) is absolutely convergent for $\Re(s)<-\sigma$.)

Equivalently the functions $H\left(\boldsymbol{q} ; c, \chi^{*}, s\right)$ and $G\left(\boldsymbol{q} ; c, \chi^{*}, s\right)$ as defined by the formulas (9) and (10) have analytic continuations to all $s$ and equal each other as in (11).

Remark 1.4 (the structure of this article). Theorem 1.3 is our main result. For the most part our focus is on the case $N \geq 3$, and we deal with the case $N=2$ in Remark 3.2. The Voronoi formula (8) is proved to be equivalent to a formula (11) involving Gauss sums. The equivalence is shown in Proposition 3.5. A convolved version of (11) is obtained in Theorem 3.1 by comparing Dirichlet coefficients of two different expressions of a double Dirichlet series. We later show in Proposition 3.3 that this convolved version yields (11).

Remark 1.5. If we start with an $L$-series $L(s, F)$ with an Euler product

$$
L(s, F)=\sum_{n=1}^{\infty} \frac{A(1, \ldots, 1, n)}{n^{s}}=\prod_{p} \prod_{i=1}^{N}\left(1-\frac{\alpha_{i}(p)}{p^{s}}\right)^{-1}
$$

and with $\prod_{i} \alpha_{i}(p)=1$ for any $p$, then we can define $A\left(p^{k_{1}}, \ldots, p^{k_{N-1}}\right)$ by the Casselman-Shalika formula [Zhou 2014, Proposition 5.1] and they are compatible with the Hecke relations. More explicitly, for a prime number $p$, we define $A\left(p^{k_{1}}, \ldots, p^{k_{N-1}}\right)=S_{k_{1}, \ldots, k_{N-1}}\left(\alpha_{1}(p), \ldots, \alpha_{N}(p)\right)$ by the work of Shintani, where 
$S_{k_{1}, \ldots, k_{N-1}}\left(x_{1}, \ldots, x_{N}\right)$ is the Schur polynomial, which can be found in [Goldfeld 2006, p. 233].

We extend the definition to all $A(*, \ldots, *)$ multiplicatively by (2). One can prove that $A(*, \ldots, *)$ satisfies the Hecke relations (2)-(4). In summary, the "coefficients" $A(*, \ldots, *)$ along with the Hecke relations can be generated by an $L$-function with an Euler product.

The following examples satisfy the conditions in Theorem 1.3, and hence we have a Voronoi formula for each of them.

Example 1.6 (automorphic form for $\operatorname{SL}(N, \mathbb{Z})$ ). Any cuspidal automorphic form for $\operatorname{SL}(N, \mathbb{Z})$ satisfies the conditions in Theorem 1.3. It can have an unramified or ramified component at the archimedean place, because only the exact form of the $G_{ \pm}$function would change; see [Godement and Jacquet 1972]. The Hecke-Maass cusp forms considered in Theorem 1.1 are included in this category, and therefore, we prove Theorem 1.3 instead of Theorem 1.1.

Example 1.7 (Rankin-Selberg convolution). Let $F_{1}$ and $F_{2}$ be even Hecke-Maass cusp forms for $\operatorname{SL}\left(N_{1}, \mathbb{Z}\right)$ and $\operatorname{SL}\left(N_{2}, \mathbb{Z}\right)$ with the spectral parameters

$$
\left(\lambda_{1}, \ldots, \lambda_{N_{1}}\right) \in \mathbb{C}^{N_{1}} \text { and }\left(\mu_{1}, \ldots, \mu_{N_{2}}\right) \in \mathbb{C}^{N_{2}}
$$

respectively. Assume $F_{1} \neq \widetilde{F}_{2}$ if $N_{1}=N_{2}$. The automorphic forms $F_{1}$ and $F_{2}$ have the standard $L$-functions

$$
L\left(s, F_{1}\right)=\prod_{p} \prod_{i=1}^{N_{1}}\left(1-\frac{\alpha_{i}(p)}{p^{s}}\right)^{-1} \quad \text { and } \quad L\left(s, F_{2}\right)=\prod_{p} \prod_{i=1}^{N_{2}}\left(1-\frac{\beta_{i}(p)}{p^{s}}\right)^{-1} \text {. }
$$

Let $L\left(s, F_{1} \times F_{2}\right)$ be the Rankin-Selberg $L$-function of $F_{1}$ and $F_{2}$ defined by

$$
L\left(s, F_{1} \times F_{2}\right)=\prod_{p} \prod_{i_{1}=1}^{N_{1}} \prod_{i_{2}=1}^{N_{2}}\left(1-\frac{\alpha_{i_{1}}(p) \beta_{i_{2}}(p)}{p^{s}}\right)^{-1} .
$$

The $L$-function is of degree $N:=N_{1} N_{2}$. The work of Jacquet, Piatetskii-Shapiro, and Shalika [Jacquet et al. 1983] shows that $L\left(s, F \times \chi^{*}\right)=L\left(s,\left(F_{1} \times \chi^{*}\right) \times F_{2}\right)$ is holomorphic and satisfies the functional equation (15) for $F:=F_{1} \times F_{2}$.

Define $A\left(p^{k_{1}}, \ldots, p^{k_{N-1}}\right)$ by the Schur polynomials as in Remark 1.5: $A\left(p^{k_{1}}, \ldots, p^{k_{N-1}}\right):=$

$$
S_{k_{1}, \ldots, k_{N-1}}\left(\alpha_{1}(p) \beta_{1}(p), \ldots, \alpha_{i_{1}}(p) \beta_{i_{2}}(p), \ldots, \alpha_{N_{1}}(p) \beta_{N_{2}}(p)\right) .
$$


Extend the definition to all $A(*, \ldots, *)$ multiplicatively by (2). Define

$$
\begin{aligned}
& G_{ \pm}(s):= \\
& \quad i^{-N \delta} \pi^{-N(1 / 2-s)} \prod_{i_{1}=1}^{N_{1}} \prod_{i_{2}=1}^{N_{2}} \Gamma\left(\frac{\delta+1-s-\overline{\lambda_{i_{1}}}-\overline{\mu_{i_{2}}}}{2}\right) \Gamma\left(\frac{\delta+s-\lambda_{i_{1}}-\mu_{i_{2}}}{2}\right)^{-1},
\end{aligned}
$$

where one takes $\delta=0$ and $\delta=1$ for $G_{+}$and $G_{-}$, respectively. Theorem 1.3 gives us a Voronoi formula for the Rankin-Selberg convolution $F=F_{1} \times F_{2}$ with the $A(*, \ldots, *)$ and $G_{ \pm}$defined above.

Example 1.8 (isobaric sum, Eisenstein series). For $i=1, \ldots, k$, let $F_{i}$ be a HeckeMaass cusp form for $\operatorname{SL}\left(N_{i}, \mathbb{Z}\right)$. Let $s_{i}$ be complex numbers with $\sum_{i} N_{i} s_{i}=0$. Define the isobaric sum $F=\left(F_{1} \times|\cdot|_{\mathbb{A}}^{s_{1}}\right) \boxplus\left(F_{2} \times|\cdot|_{\AA}^{s_{2}}\right) \boxplus \cdots \boxplus\left(F_{k} \times|\cdot|_{A}^{s_{k}}\right)$, whose $L$-function is $L(s, F)=\prod_{i} L\left(s+s_{i}, F_{i}\right)$. This isobaric sum $F$ is associated with a noncuspidal automorphic form on $\mathrm{GL}(N)$, an Eisenstein series twisted by Maass forms, where $N=\sum_{i} N_{i}$; see [Goldfeld 2006, Section 10.5]. The $L$-function twisted by a character is simply given by $L\left(s, F \times \chi^{*}\right)=\prod_{i} L\left(s+s_{i}, F_{i} \times \chi^{*}\right)$, which satisfies the conditions of Theorem 1.3.

Example 1.9 (symmetric powers on GL(2)). Let $f$ be a modular form of weight $k$ for $\operatorname{SL}(2, \mathbb{Z})$, and define $F:=\operatorname{Sym}^{2} f$. The symmetric square $F$ satisfies the conditions in Theorem 1.3 by the work of Shimura [1975]. Here we do not need to involve automorphy using Gelbart-Jacquet lifting. One may have similar results for higher symmetric powers depending on the recent progress in the theory of Galois representations.

As a last remark, let us explain the construction of the double Dirichlet series $Z(s, w)$ given by (12). This construction originates from the Rankin-Selberg convolution of a cusp form $F$ and an Eisenstein series on GL(2). The Fourier coefficients of the Eisenstein series $E\left(z, s, \chi^{*}\right)$ can be written in terms of the divisor function $\sigma_{2 s-1}\left(n, \chi^{*}\right)$ defined in Definition 2.1:

$$
\frac{1}{n^{2 s-1}} \frac{\sigma_{2 s-1}\left(n, \chi^{*}\right)}{L\left(2 s, \overline{\chi^{*}}\right)} \quad \text { or } \sum_{\ell=1}^{\infty} \frac{g\left(\overline{\chi^{*}}, \ell c^{*}, n\right)}{\left(\ell c^{*}\right)^{2 s}} .
$$

Therefore, in the case of $F$ on GL(2), the Rankin-Selberg integral of $F$ and $E\left(*, w-s+\frac{1}{2}, \chi^{*}\right)$ produces the double Dirichlet series

$$
\sum_{n=1}^{\infty} \sum_{\ell=1}^{\infty} \frac{A(n) g\left(\overline{\chi^{*}}, \ell c^{*}, n\right)}{n^{s}\left(\ell c^{*}\right)^{2 w+1-2 s}} .
$$

A similar expression appears on the left-hand side of the Voronoi formula with Gauss sums (9). The Rankin-Selberg convolution of the cusp form $F$ and an 
Eisenstein series can be written as a product of two copies of a standard $L$-function of $F$, namely

$$
\frac{L(2 w-s, F) L\left(s, F \times \chi^{*}\right)}{L\left(2 w-2 s+1, \overline{\chi^{*}}\right)} .
$$

Applying the functional equation to only $L\left(s, F \times \chi^{*}\right)$ gives us another expression, which is similar to the right-hand side (10) of the Voronoi formula with Gauss sums. Since $L(2 w-s, F)$ was not used in this process, we have the freedom to replace $L(2 w-s, F)$ by $L_{\boldsymbol{q}}(2 w-s, F)$ in the case of GL $(N)$, and it gives us enough generality to prove the Voronoi formula (11) with Gauss sums. In the case of GL(3), this construction is similar to Bump's double Dirichlet series; see [Goldfeld 2006, Chapter 6.6] or [Bump 1984, Chapter X].

\section{Background on Gauss sums}

Here we collect information about the Gauss sums of Dirichlet characters which are not necessarily primitive.

Definition 2.1. Let $\chi$ be a Dirichlet character modulo $c$ induced from a primitive Dirichlet character $\chi^{*}$ modulo $c^{*}$. Define the divisor function

$$
\sigma_{s}(m, \chi)=\sum_{d \mid m} \chi(d) d^{s} .
$$

Define the Gauss sum of $\chi$ to be

$$
g\left(\chi^{*}, c, m\right)=\sum_{\substack{(u, c)=1 \\ u \bmod c}} \chi(u) e\left(\frac{m u}{c}\right) .
$$

The standard Gauss sum for $\chi^{*}$ is given as $\tau\left(\chi^{*}\right)=g\left(\chi^{*}, c^{*}, 1\right)$.

The Gauss sum $g\left(\chi^{*}, c, m\right)$ is the same as the Gauss sum $\tau_{m}(\chi)$ in other literature. However we prefer our notation because we come upon numerous Gauss sums of characters $\chi$ induced from a single primitive character $\chi^{*}$.

Lemma 2.2 (Gauss sum of nonprimitive characters [Miyake 1989, Lemma 3.1.3(2)]). Let $\chi$ be a character modulo $c$ induced from a primitive character $\chi^{*}$ modulo $c^{*}$. Then the Gauss sum of $\chi$ is given by

$$
g\left(\chi^{*}, c, a\right)=\tau\left(\chi^{*}\right) \sum_{d \mid\left(a, c / c^{*}\right)} d \chi^{*}\left(\frac{c}{c^{*} d}\right) \overline{\chi^{*}}\left(\frac{a}{d}\right) \mu\left(\frac{c}{c^{*} d}\right) .
$$

Lemma 2.3 [Montgomery and Vaughan 2007, Theorem 9.12]. Let $\chi^{*}$ be a primitive character modulo $c^{*}$ and assume $c^{*} \mid c$. Then we have

$$
g\left(\chi^{*}, c, a\right)=\tau\left(\chi^{*}\right) \frac{\phi(c)}{\phi(c /(c, a))} \mu\left(\frac{c}{c^{*}(c, a)}\right) \chi^{*}\left(\frac{c}{c^{*}(c, a)}\right) \overline{\chi^{*}}\left(\frac{a}{(c, a)}\right)
$$

if $c^{*} \mid c /(a, c)$. Otherwise, $g\left(\chi^{*}, c, a\right)$ is zero. 
The next lemma is a generalization of a famous formula of Ramanujan:

$$
\frac{\sigma_{s-1}(n)}{n^{s-1}}=\zeta(s) \sum_{\ell=1}^{\infty} \frac{c_{\ell}(n)}{\ell^{s}},
$$

where $c_{\ell}(n)$ is the Ramanujan sum.

Lemma 2.4. Let $\Re(s)>1$. Define a Dirichlet series

$$
I\left(s, \chi^{*}, c^{*}, m\right)=\sum_{\ell=1}^{\infty} \frac{g\left(\chi^{*}, \ell c^{*}, m\right)}{\ell^{s}}
$$

as a generating function for the nonprimitive Gauss sums induced from $\chi^{*}$. It satisfies the identity

$$
\tau\left(\chi^{*}\right) \sigma_{s-1}\left(m, \overline{\chi^{*}}\right)=m^{s-1} I\left(s, \chi^{*}, c^{*}, m\right) L\left(s, \chi^{*}\right) .
$$

Proof. We prove the equivalent formula

$$
\tau\left(\chi^{*}\right) m^{1-s} \sigma_{s-1}\left(m, \overline{\chi^{*}}\right) L\left(s, \chi^{*}\right)^{-1}=I\left(s, \chi^{*}, c^{*}, m\right) .
$$

For $\Re(s)>1$, the function $\tau\left(\chi^{*}\right) m^{1-s} \sigma_{s-1}\left(m, \overline{\chi^{*}}\right) L\left(s, \chi^{*}\right)^{-1}$ can be written as a Dirichlet series

$$
\begin{aligned}
\tau\left(\chi^{*}\right) \sum_{d \mid m} \frac{d \overline{\chi^{*}}(m / d)}{d^{s}} \sum_{n=1}^{\infty} \frac{\chi^{*}(n) \mu(n)}{n^{s}} & \\
& =\tau\left(\chi^{*}\right) \sum_{\ell=1}^{\infty} \frac{\sum_{d \mid(m, \ell)} d \overline{\chi^{*}}(m / d) \mu(\ell / d) \chi^{*}(\ell / d)}{\ell^{s}},
\end{aligned}
$$

and this equals $I\left(s, \chi^{*}, c^{*}, m\right)$ by Lemma 2.2.

Lemma 2.5. For any two positive integers $n$ and $m$, and a primitive Dirichlet character $\chi^{*}$ modulo $c^{*}$, we have

$$
\sum_{\ell d=n} \chi^{*}(d) g\left(\chi^{*}, \ell c^{*}, m\right)=\left\{\begin{array}{cl}
\tau\left(\chi^{*}\right) \overline{\chi^{*}(m / n)} n & \text { if } n \mid m, \\
0 & \text { otherwise. }
\end{array}\right.
$$

Proof. We start with the formula,

$$
\frac{\tau\left(\chi^{*}\right) \sigma_{s-1}\left(m, \overline{\chi^{*}}\right)}{m^{s-1}}=I\left(s, \chi^{*}, c^{*}, m\right) L\left(s, \chi^{*}\right) .
$$

Both sides are Dirichlet series and we equate coefficients. The left-hand side is given as

$$
\tau\left(\chi^{*}\right) \sum_{e \mid m} \frac{\overline{\chi^{*}(m / e)} e}{e^{s}}
$$


whereas the right-hand side is

$$
\sum_{\ell=1}^{\infty} \frac{g\left(\chi^{*}, \ell c^{*}, m\right)}{\ell^{s}} \sum_{d=1}^{\infty} \frac{\chi^{*}(d)}{d^{s}}=\sum_{n=1}^{\infty} \frac{\sum_{d \ell=n} \chi^{*}(d) g\left(\chi^{*}, \ell c^{*}, m\right)}{n^{s}} .
$$

\section{The Voronoi formula}

3A. Double Dirichlet series. We begin by proving a convolved version of (11).

Theorem 3.1. For $N \geq 3, \boldsymbol{q}=\left(q_{1}, \ldots, q_{N-2}\right) \in \mathbb{N}^{N-2}$, and $n \in \mathbb{N}$, define

$$
\mathscr{H}(\boldsymbol{q} ; n, s):=\sum_{d_{1}\left|q_{1}, \ldots, d_{N-2}\right| q_{N-2}} \frac{\chi^{*}\left(d_{1} \cdots d_{N-2}\right)}{\left(d_{1} \cdots d_{N-2}\right)^{s}} \sum_{d \ell=n} \chi^{*}(d) H\left(\boldsymbol{q}^{\prime} ; \ell c^{*}, \chi^{*}, s\right)
$$

for $\Re(s) \gg 1$, and

$$
\mathscr{G}(\boldsymbol{q} ; n, s):=\sum_{d_{1}\left|q_{1}, \ldots, d_{N-2}\right| q_{N-2}} \frac{\chi^{*}\left(d_{1} \cdots d_{N-2}\right)}{\left(d_{1} \cdots d_{N-2}\right)^{s}} \sum_{d \ell=n} \chi^{*}(d) G\left(\boldsymbol{q}^{\prime} ; \ell c^{*}, \chi^{*}, s\right)
$$

for $\Re(1-s) \gg 1$, where we abbreviate

$$
\boldsymbol{q}^{\prime}=\left(\frac{q_{1} d}{d_{1}}, \frac{q_{2} d_{1}}{d_{2}}, \ldots, \frac{q_{N-2} d_{N-3}}{d_{N-2}}\right) .
$$

The functions $\mathcal{H}(\boldsymbol{q} ; n, s)$ and $\mathscr{G}(\boldsymbol{q} ; n, s)$ have analytic continuation to all $s \in \mathbb{C}$ and these analytic continuations satisfy

$$
\mathscr{H}(\boldsymbol{q} ; n, s)=\mathscr{G}(\boldsymbol{q} ; n, s) .
$$

Proof. The region of absolute convergence for $\mathscr{H}(\boldsymbol{q} ; n, s)$ is a right half plane $\Re(s) \gg 1$, and the region of absolute convergence of $\mathscr{G}(\boldsymbol{q} ; n ; s)$ is a left half plane $\Re(1-s) \gg 1$. Let $Z(s, w)$ be defined as in (12). For any $s \in \mathbb{C}$ and $w$ with $\Re(w)$ large enough so that $\Re(2 w-s) \gg 1$ and $\Re(w-s)>0$, writing $L_{\boldsymbol{q}}(2 w-s, F)$ and $L\left(2 w-2 s+1, \overline{\chi^{*}}\right)^{-1}$ as Dirichlet series, we derive

$Z(s, w)=L\left(s, F \times \chi^{*}\right) \sum_{n=1}^{\infty} \frac{\sum_{d \mid n} A\left(q_{N-2}, \ldots, q_{1}, d\right) d^{s} \overline{\chi^{*}}(n / d) \mu(n / d)(n / d)^{2 s-1}}{n^{2 w}}$.

Hence, we have

$$
Z(s, w)=\sum_{n=1}^{\infty} \frac{a_{n}(s)}{n^{2 w}}
$$

where

$$
a_{n}(s)=L\left(s, F \times \chi^{*}\right) \sum_{d \mid n} A\left(q_{N-2}, \ldots, q_{1}, d\right) d^{s} \overline{\chi^{*}}(n / d) \mu(n / d)(n / d)^{2 s-1} .
$$

Here $a_{n}(s)$ is an analytic function of $s \in \mathbb{C}$, because $L\left(s, F \times \chi^{*}\right)$ is entire. The computation below shows that $a_{n}(s)$ equals either side of (17) in their respective 
regions of absolute convergence, up to scaling by a constant $\tau\left(\overline{\chi^{*}}\right)$. This proves the analytic continuation of $\mathscr{H}$ and $\mathscr{G}$ as well as their equality.

For $\Re(s) \gg 1, \Re(w-s)>0$, we expand the two $L$-functions in the numerator of $Z(s, w)$ as Dirichlet series, obtaining

$$
\begin{aligned}
& Z(s, w)= \frac{1}{L\left(2 w-2 s+1, \overline{\chi^{*}}\right)} \sum_{n, m=1}^{\infty} \frac{A\left(q_{N-2}, \ldots, q_{1}, n\right) A(1, \ldots, 1, m) \chi^{*}(m)}{n^{2 w-s} m^{s}} \\
&= \frac{1}{L\left(2 w-2 s+1, \overline{\chi^{*}}\right)} \sum_{n, m=1}^{\infty}\left(\frac{\chi^{*}(m)}{n^{2 w-s} m^{s}}\right. \\
&\left.\times \sum_{\substack{d_{0} d_{1} \cdots d_{N-1}=m \\
d_{0}\left|n, d_{1}\right| q_{1}, \ldots, d_{N-2} \mid q_{N-2}}} A\left(\frac{q_{N-2} d_{N-3}}{d_{N-2}}, \ldots, \frac{q_{1} d_{0}}{d_{1}}, \frac{n d_{N-1}}{d_{0}}\right)\right),
\end{aligned}
$$

where we have used the Hecke relation (3). We change the variable $n / d_{0} \rightarrow n$ and combine $h=n d_{N-1}$, giving

$$
\begin{array}{r}
Z(s, w)=\frac{1}{L\left(2 w-2 s+1, \overline{\chi^{*}}\right)} \sum_{n, d_{0}, d_{N-1}=1}^{\infty} \sum_{\substack{d_{i} \mid q_{i} \\
i=1, \ldots, N-2}} \frac{\chi^{*}\left(d_{0} \cdots d_{N-1}\right)}{n^{2 w-s} d_{0}^{2 w-s}\left(d_{0} \cdots d_{N-1}\right)^{s}} \\
\quad \times A\left(\frac{q_{N-2} d_{N-3}}{d_{N-2}}, \ldots, \frac{q_{1} d_{0}}{d_{1}}, n d_{N-1}\right) \\
=\frac{1}{L\left(2 w-2 s+1, \overline{\chi^{*}}\right)} \sum_{d_{0}, h=1}^{\infty} \sum_{\substack{d_{i} \mid q_{i} \\
i=1, \ldots, N-2}} \frac{\chi^{*}\left(d_{0} \cdots d_{N-2}\right)}{d_{0}^{2 w-s}\left(d_{0} \cdots d_{N-2}\right)^{s}} \\
\times A\left(\frac{q_{N-2} d_{N-3}}{d_{N-2}}, \ldots, \frac{q_{1} d_{0}}{d_{1}}, h\right) \frac{\sigma_{2 w-2 s}\left(h, \chi^{*}\right)}{h^{2 w-s}} .
\end{array}
$$

Applying Lemma 2.4, we get

$$
\begin{aligned}
Z(s, w)=\tau\left(\overline{\chi^{*}}\right)^{-1} \sum_{d_{0}=1}^{\infty} & \sum_{\substack{d_{i} \mid q_{i} \\
i=1, \ldots, N-2}}\left(\frac{\chi^{*}\left(d_{0} \cdots d_{N-2}\right)}{d_{0}^{2 w}\left(d_{1} \cdots d_{N-2}\right)^{s}}\right. \\
& \left.\times \sum_{h=1}^{\infty} \frac{1}{h^{s}} A\left(\frac{q_{N-2} d_{N-3}}{d_{N-2}}, \ldots, \frac{q_{1} d_{0}}{d_{1}}, h\right) \sum_{\ell=1}^{\infty} \frac{g\left(\overline{\chi^{*}}, \ell c^{*}, h\right)}{\ell^{2 w-2 s+1}}\right) .
\end{aligned}
$$

Therefore, defining $\boldsymbol{q}^{\prime}$ as in (16), we reach

$$
\begin{aligned}
Z(s, w)=\tau\left(\overline{\chi^{*}}\right)^{-1} \sum_{n=1}^{\infty} \frac{1}{n^{2 w}} \sum_{d_{1}\left|q_{1}, \ldots, d_{N-2}\right| q_{N-2}} & \left(\frac{\chi^{*}\left(d_{1} \cdots d_{N-2}\right)}{\left(d_{1} \cdots d_{N-2}\right)^{s}}\right. \\
& \left.\times \sum_{d \ell=n} \chi^{*}(d) H\left(\boldsymbol{q}^{\prime} ; \ell c^{*}, \chi^{*}, s\right)\right) .
\end{aligned}
$$


On the other hand, let us apply the functional (15) to $L\left(s, F \times \chi^{*}\right)$ in $Z(s, w)$, giving

$$
Z(s, w)=\frac{G(s) \tau\left(\chi^{*}\right)^{N}}{c^{* N s}} \frac{L_{\boldsymbol{q}}(2 w-s, F) L\left(1-s, \widetilde{F} \times \overline{\chi^{*}}\right)}{L\left(2 w-2 s+1, \overline{\chi^{*}}\right)} .
$$

Given $\Re(1-s) \gg 1$ and $\Re(2 w-s) \gg 1$, we open the expression as a Dirichlet series:

$$
\begin{aligned}
& Z(s, w) \\
& =\frac{G(s) \tau\left(\chi^{*}\right)^{N} c^{*-N s}}{L\left(2 w-2 s+1, \overline{\chi^{*}}\right)} \sum_{n, m=1}^{\infty} \frac{A\left(q_{N-2}, \ldots, q_{1}, n\right) A(m, 1, \ldots, 1) \overline{\chi^{*}}(m)}{n^{2 w-s} m^{1-s}} \\
& =\frac{G(s) \tau\left(\chi^{*}\right)^{N} c^{*-N s}}{L\left(2 w-2 s+1, \overline{\chi^{*}}\right)} \\
& \times \sum_{n, m=1}^{\infty} \frac{\overline{\chi^{*}}(m)}{n^{2 w-s} m^{1-s}} \sum_{\begin{array}{c}
d_{0} d_{1} \cdots d_{N-1}=m \\
d_{0}\left|n, d_{1}\right| q_{1}, \ldots, d_{N-2} \mid q_{N-2}
\end{array}} A\left(\frac{q_{N-2} d_{N-1}}{d_{N-2}}, \ldots, \frac{q_{1} d_{2}}{d_{1}}, \frac{n d_{1}}{d_{0}}\right) \\
& =\frac{G(s) \tau\left(\chi^{*}\right)^{N} c^{*-N s}}{L\left(2 w-2 s+1, \overline{\chi^{*}}\right)} \\
& \times \sum_{n, m=1}^{\infty} \sum_{\substack{d_{0} d_{1} \cdots d_{N-1}=m \\
d_{0}\left|n, d_{1}\right| q_{1}, \ldots, d_{N-2} \mid q_{N-2}}} \\
& \frac{\overline{\chi^{*}}\left(d_{0} d_{1} \cdots d_{N-1}\right) A\left(\frac{q_{N-2} d_{N-1}}{d_{N-2}}, \ldots, \frac{q_{1} d_{2}}{d_{1}}, \frac{n d_{1}}{d_{0}}\right)}{\left(n / d_{0}\right)^{2 w-s} d_{0}^{1+2 w-2 s}\left(d_{1} \cdots d_{N-1}\right)^{1-s}},
\end{aligned}
$$

where we have combined the Fourier coefficients by the Hecke relation (4). We change the variable $n / d_{0} \rightarrow n$. Then the sum over $d_{0}$ cancels with $L\left(2 w-2 s+1, \overline{\chi^{*}}\right)$ in the denominator, giving

$$
\begin{aligned}
& Z(s, w) \\
& \begin{array}{r}
\frac{G(s) \tau\left(\chi^{*}\right)^{N} c^{*-N s}}{L\left(2 w-2 s+1, \overline{\chi^{*}}\right)} \sum_{n, d_{0}, d_{N-1}=1}^{\infty} \sum_{\substack{d_{i} \mid q_{i} \\
i=1, \ldots, N-2}} A\left(\frac{q_{N-2} d_{N-1}}{d_{N-2}}, \ldots, \frac{q_{1} d_{2}}{d_{1}}, d_{1} n\right) \\
\times \frac{\overline{\chi^{*}}\left(d_{0} d_{1} \cdots d_{N-1}\right)}{n^{2 w-s} d_{0}^{1+2 w-2 s}\left(d_{1} \cdots d_{N-1}\right)^{1-s}} \\
=\frac{G(s) \tau\left(\chi^{*}\right)^{N}}{c^{* N s}} \sum_{n, d_{N-1}=1}^{\infty} \sum_{\substack{d_{i} \mid q_{i} \\
i=1, \ldots, N-2}} \frac{\overline{\chi^{*}}\left(d_{1} \cdots d_{N-1}\right)}{n^{2 w-s}\left(d_{1} \cdots d_{N-1}\right)^{1-s}} .
\end{array}
\end{aligned}
$$

If we denote the right-hand side of (17) by $\tau\left(\overline{\chi^{*}}\right) b_{n}(s)$, our goal is to transform (19) into $R:=\sum_{n=1}^{\infty} b_{n}(s) n^{-2 w}$. But at this point it is easier to start from $R$. More explicitly, we have 


$$
\begin{gathered}
R=\tau\left(\overline{\chi^{*}}\right)^{-1} \sum_{h=1}^{\infty} \frac{1}{h^{2 w}} \sum_{d_{1}\left|q_{1}, \ldots, d_{N-2}\right| q_{N-2}}\left(\frac{\chi^{*}\left(d_{1} \cdots d_{N-2}\right)}{\left(d_{1} \cdots d_{N-2}\right)^{s}}\right. \\
\left.\times \sum_{d \ell=h} \chi^{*}(d) G\left(\boldsymbol{q}^{\prime} ; \ell c^{*}, \chi^{*}, s\right)\right) .
\end{gathered}
$$

Here $\boldsymbol{q}^{\prime}$ has been defined in (16). We plug in the definition of $G\left(\boldsymbol{q}^{\prime} ; \ell c^{*}, \chi^{*}, s\right)$ from (10) for $\boldsymbol{q}^{\prime}$, giving

$$
\begin{gathered}
G\left(\boldsymbol{q}^{\prime} ; \ell c^{*}, \chi^{*}, s\right) \\
=\frac{G(s) \chi^{*}(-1)}{c^{* N s-1} \ell^{(N-2) s}} \sum_{f_{1} \mid \frac{q_{1} d \ell}{d_{1}}} \sum_{f_{2} \mid \frac{q_{1} q_{2} d \ell}{f_{1} d_{2}}} \ldots \sum_{f_{N-2} \mid \frac{q_{1} \cdots q_{N-2} d \ell}{f_{1} \cdots f_{N-3} d_{N-2}}} \\
\quad \sum_{n=1}^{\infty} \frac{A\left(n, f_{N-2}, \ldots, f_{1}\right)}{n^{1-s} f_{1} f_{2} \cdots f_{N-2}} \frac{f_{1}^{(N-1) s} f_{2}^{(N-2) s} \cdots f_{N-2}^{2 s}}{q_{1}^{(N-2) s} q_{2}^{(N-3) s} \cdots q_{N-2}^{s}} \frac{\left(d_{1} \cdots d_{N-2}\right)^{s}}{d^{(N-2) s}} \\
\quad \times g\left(\chi^{*}, \ell c^{*}, f_{1}\right) g\left(\chi^{*}, \frac{q_{1} d \ell c^{*}}{f_{1} d_{1}}, f_{2}\right) \\
\quad \cdots \times g\left(\chi^{*}, \frac{q_{1} \cdots q_{N-3} d \ell c^{*}}{f_{1} \cdots f_{N-3} d_{N-3}}, f_{N-2}\right) g\left(\chi^{*}, \frac{q_{1} \cdots q_{N-2} d \ell c^{*}}{f_{1} \cdots f_{N-2} d_{N-2}}, n\right) .
\end{gathered}
$$

We substitute $G\left(\boldsymbol{q}^{\prime} ; \ell c^{*}, \chi^{*}, s\right)$ with this expression in (20) and change the orders of summation between $f_{i}$ and $d_{i}$. The summations over $d$ and $d_{i}$ collapse with the repeated use of Lemma 2.5, giving

$$
\begin{gathered}
R=\tau\left(\overline{\chi^{*}}\right)^{-1} \frac{G(s) \chi^{*}(-1)}{c^{* N s-1}} \sum_{h=1}^{\infty} \sum_{n=1}^{\infty} \sum_{\substack{h\left|f_{1} \\
f_{1}\right| q_{1} h}} \sum_{\substack{q_{1} h \\
f_{1}\left|f_{2} \\
f_{2}\right| \frac{q_{1} q_{2} h}{f_{1}}}} \ldots \sum_{\substack{q_{1} \cdots q_{N-3} h \\
f_{1} \cdots f_{N-3} \\
f_{N-2} \mid \frac{q_{1} \cdots q_{N-2} h}{f_{1} \cdots f_{N-3}}}} \sum_{\substack{q_{1} \cdots q_{N-2} h \\
f_{1} \cdots f_{N-2}}} \frac{\tau\left(\chi^{*}\right)^{N-1}}{h^{2 w}} \\
\times \overline{\chi^{*}}\left(\frac{f_{1}}{h}\right) \overline{\chi^{*}}\left(\frac{f_{1} f_{2}}{h q_{1}}\right) \cdots \overline{\chi^{*}}\left(\frac{f_{1} f_{2} \cdots f_{N-2}}{h q_{1} \cdots q_{N-3}}\right) \overline{\chi^{*}}\left(\frac{f_{1} f_{2} \cdots f_{N-2} n}{h q_{1} \cdots q_{N-2}}\right) \\
\times\left(\frac{q_{1}}{f_{1}}\right)^{N-2}\left(\frac{q_{2}}{f_{2}}\right)^{N-3} \cdots\left(\frac{q_{N-2}}{f_{N-2}}\right) h^{N-1-N s+2 s} \\
\times \frac{A\left(n, f_{N-2}, \ldots, f_{1}\right)}{n^{1-s} f_{1} \cdots f_{N-2}} \frac{f_{1}^{(N-1) s} \cdots f_{N-2}^{2 s}}{q_{1}^{(N-2) s} \cdots q_{N-2}^{s}} .
\end{gathered}
$$

Define $e_{1}=f_{1} / h$ and $e_{i}=\left(f_{1} \cdots f_{i}\right) /\left(q_{1} \cdots q_{i-1} h\right)$ for $i=2, \ldots, N-2$, so that the double conditions under the sums simplify to $e_{i} \mid q_{i}$. Extend this to all positive integers by setting $e_{N-1}=\left(f_{1} \cdots f_{N-2} n\right) /\left(h q_{1} \cdots q_{N-2}\right)$. Finally, noting $\tau\left(\overline{\chi^{*}}\right)^{-1}=\chi^{*}(-1) \tau\left(\chi^{*}\right) / c^{*}$, we get 


$$
\begin{aligned}
R=\frac{G(s) \tau\left(\chi^{*}\right)^{N}}{c^{* N s}} \sum_{h, e_{N-1}=1}^{\infty} \frac{1}{h^{2 w-s}} \sum_{\substack{e_{i} \mid q_{i} \\
i=1, \ldots, N-2}} \frac{\overline{\chi^{*}\left(e_{1} \cdots e_{N-2} e_{N-1}\right)}}{\left(e_{1} \cdots e_{N-1}\right)^{1-s}} \\
\quad \times A\left(\frac{e_{N-1} q_{N-2}}{e_{N-2}}, \ldots, \frac{e_{2} q_{1}}{e_{1}}, e_{1} h\right),
\end{aligned}
$$

which in turn, by (19), equals $Z(s, w)$ as well as (18). We complete the proof by applying the uniqueness theorem for Dirichlet series [Apostol 1976, Theorem 11.3] to the equality between (18) and (20).

Remark 3.2. The above proof works for $N \geq 3$ but not for $N=2$. We can prove the Voronoi formula for $\operatorname{SL}(2, \mathbb{Z})$ similarly and easily by considering

$$
Z(s, w)=\frac{L(2 w-s, F) L\left(s, F \times \chi^{*}\right)}{L\left(2 w-2 s+1, \overline{\chi^{*}}\right) L\left(2 w, \chi^{*}\right)} .
$$

We have, from the Hecke relations on GL(2),

$$
Z(s, w)=\tau\left(\overline{\chi^{*}}\right)^{-1} \sum_{\ell=1}^{\infty} \sum_{n=1}^{\infty} \frac{A(n)}{n^{s}} \frac{g\left(\overline{\chi^{*}}, \ell c^{*}, n\right)}{\ell^{1+2 w-2 s}},
$$

and applying the functional equation for $L\left(s, F \times \chi^{*}\right)$ we have

$$
Z(s, w)=\tau\left(\chi^{*}\right) c^{*-2 s} G(s) \sum_{\ell=1}^{\infty} \sum_{n=1}^{\infty} \frac{A(n)}{n^{1-s}} \frac{g\left(\chi^{*}, \ell c^{*}, n\right)}{\ell^{2 w}} .
$$

Applying the uniqueness theorem for Dirichlet series to the variable $w$, we get the Voronoi formula with Gauss sums on GL(2).

Proposition 3.3. Equation (11) is equivalent to Theorem 3.1.

Proof. Construct the following summation:

$$
\begin{aligned}
& T:=\sum_{e_{0} \mid n} \sum_{e_{1} \mid q_{1} e_{0}} \cdots \sum_{e_{N-2} \mid q_{N-2} e_{N-3}} \frac{\mu\left(e_{0} \cdots e_{N-2}\right) \chi^{*}\left(e_{0} \cdots e_{N-2}\right)}{\left(e_{1} \cdots e_{N-2}\right)^{s}} \\
& \times \mathscr{H}\left(\frac{q_{1} e_{0}}{e_{1}}, \ldots, \frac{q_{N-2} e_{N-3}}{e_{N-2}} ; \frac{n}{e_{0}}, s\right) \\
& =\sum_{e_{0} \mid n} \sum_{e_{1} \mid q_{1} e_{0}} \cdots \sum_{e_{N-2} \mid q_{N-2} e_{N-3}}\left(\frac{\mu\left(e_{0} \cdots e_{N-2}\right) \chi^{*}\left(e_{0} \cdots e_{N-2}\right)}{\left(e_{1} \cdots e_{N-2}\right)^{s}}\right. \\
& \times \sum_{\substack{d_{i} \mid q_{i} e_{i-1} / e_{i} \\
i=1, \ldots, N-2}} \frac{\chi^{*}\left(d_{1} \cdots d_{N-2}\right)}{\left(d_{1} \cdots d_{N-2}\right)^{s}} \sum_{d_{0} \mid n / e_{0}} \chi^{*}\left(d_{0}\right) \\
& \left.\times H\left(\frac{q_{1} e_{0} d_{0}}{e_{1} d_{1}}, \ldots, \frac{q_{N-2} e_{N-3} d_{N-3}}{e_{N-2} d_{N-2}} ; \frac{n}{e_{0} d_{0}} c^{*}, \chi^{*}, s\right)\right) .
\end{aligned}
$$


Change variables $e_{i} d_{i} \rightarrow a_{i}$ for $i=0, \ldots, N-2$, and change orders of summation, getting

$$
\begin{aligned}
T=\sum_{a_{0} \mid n} \sum_{e_{0} \mid a_{0}} \sum_{a_{1} \mid q_{1} e_{0}} \sum_{e_{1} \mid a_{1}} \ldots \sum_{a_{N-2} \mid q_{N-2} e_{N-3}} \sum_{e_{N-2} \mid a_{N-2}} \frac{\chi^{*}\left(a_{0} \cdots a_{N-2}\right)}{\left(a_{1} \cdots a_{N-2}\right)^{s}} \\
\times H\left(\frac{q_{1} a_{0}}{a_{1}}, \frac{q_{2} a_{1}}{a_{2}}, \ldots, \frac{q_{N-2} a_{N-3}}{a_{N-2}} ; \frac{n c^{*}}{a_{0}}, \chi^{*}, s\right) \mu\left(e_{0}\right) \cdots \mu\left(e_{N-2}\right) .
\end{aligned}
$$

One by one, the Möbius summation over $e_{i}$ forces $a_{i}=1$, and thus we obtain $T=H\left(\boldsymbol{q} ; n c^{*}, \chi^{*}, s\right)$. By Theorem 3.1, we have $\mathscr{H}=\mathscr{G}$, and the same calculations yield $T=G\left(\boldsymbol{q} ; n c^{*}, \chi^{*}, s\right)$. This proves the theorem.

3B. Equivalence between equations (8) and (11). First we prove a lemma showing that the hyper-Kloosterman sum on the right-hand side of (8) becomes a product of $(N-2)$ Gauss sums after averaging against a Dirichlet character.

Lemma 3.4. Let $\chi$ be a Dirichlet character modulo $c$ which is induced from the primitive character $\chi^{*}$ modulo $c^{*}$. Let $\boldsymbol{q}=\left(q_{1}, \ldots, q_{N-2}\right)$ and $\boldsymbol{d}=\left(d_{1}, \ldots, d_{N-2}\right)$ be two tuples of positive integers, and assume that all the divisibility conditions in (1) are met. Consider the summation

$$
S:=\sum_{\substack{a \bmod c \\(a, c)=1}} \chi(a) \operatorname{Kl}(a, n, c ; \boldsymbol{q}, \boldsymbol{d})
$$

The quantity $S$ is zero unless the divisibility conditions

$$
d_{1} c^{*}\left|q_{1} c, \quad d_{2} c^{*}\right| \frac{q_{1} q_{2} c}{d_{1}}, \quad d_{3} c^{*}\left|\frac{q_{1} q_{2} q_{3} c}{d_{1} d_{2}}, \ldots, d_{N-2} c^{*}\right| \frac{q_{1} \cdots q_{N-2} c}{d_{1} \cdots d_{N-3}}
$$

are satisfied. Under such divisibility conditions, setting $\xi_{i}:=\left(q_{1} \cdots q_{i} c\right) /\left(d_{1} \cdots d_{i}\right)$, $S$ can be written as a product of Gauss sums:

$$
S=g\left(\chi^{*}, c, d_{1}\right) g\left(\chi^{*}, \xi_{1}, d_{2}\right) \cdots g\left(\chi^{*}, \xi_{N-3}, d_{N-2}\right) g\left(\chi^{*}, \xi_{N-2}, n\right) .
$$

Proof. The divisibility conditions (1) imply

$$
d_{1}\left|q_{1}\left(c, d_{1}\right), \quad d_{2}\right| q_{2}\left(\xi_{1}, d_{2}\right), \quad \ldots, \quad d_{N-2} \mid q_{N-2}\left(\xi_{N-3}, d_{N-2}\right)
$$

We open up the hyper-Kloosterman sum in $S$. The forthcoming computation is an iterative process. The summation over $a$ yields a Gauss sum, which in turn produces the term $\overline{\chi^{*}}\left(x_{1}\right)$. Then the summation over $x_{1}$ yields another Gauss sum, which produces the term $\overline{\chi^{*}}\left(x_{2}\right)$, and so on. 
First, we sum over $a$ modulo $c$ :

$$
\begin{aligned}
S & =\sum_{a \bmod c} \chi(a) \sum_{x_{1} \bmod \xi_{1}}^{*} e\left(\frac{d_{1} x_{1} a}{c}\right)\left(\sum_{x_{2} \bmod \xi_{2}}^{*} e\left(\frac{d_{2} x_{2} \overline{x_{1}}}{\xi_{1}}\right) \cdots\right) \\
& =\sum_{x_{1} \bmod \xi_{1}}^{*} g\left(\chi^{*}, c, x_{1} d_{1}\right)\left(\sum_{x_{2} \bmod \xi_{2}}^{*} e\left(\frac{d_{2} x_{2} \overline{x_{1}}}{\xi_{1}}\right) \cdots\right) .
\end{aligned}
$$

Now, because $\left(c, x_{1} d_{1}\right)=\left(\left(c, q_{1} c\right), x_{1} d_{1}\right)=\left(c,\left(q_{1} c, x_{1} d_{1}\right)\right)=\left(c, d_{1}\right)$, we deduce from Lemma 2.3 that

$$
g\left(\chi^{*}, c, x_{1} d_{1}\right)=\overline{\chi^{*}\left(x_{1}\right)} g\left(\chi^{*}, c, d_{1}\right) .
$$

By Lemma 2.3, this Gauss sum is zero unless $c^{*} \mid c /\left(c, d_{1}\right)$, which implies the first divisibility condition of (21) because, by (22),

$$
c^{*}\left|\frac{c}{\left(c, d_{1}\right)}=\frac{d_{1}}{\left(c, d_{1}\right)} \frac{c}{d_{1}}\right| \frac{q_{1} c}{d_{1}} .
$$

Next we sum over $x_{1}$. Notice that $\overline{x_{1}}$ is its multiplicative inverse modulo $q_{1} c / d_{1}$, and hence modulo $c^{*}$. This means that $\chi^{*}\left(\overline{x_{1}}\right)=\overline{\chi^{*}\left(x_{1}\right)}$. We change variables in the $x_{1}$ summation $x_{1} \rightarrow \overline{x_{1}}$, and change orders of summation to obtain

$$
\begin{aligned}
S & =g\left(\chi^{*}, c, d_{1}\right) \sum_{x_{1} \bmod \xi_{1}}^{*} \overline{\chi^{*}\left(x_{1}\right)}\left(\sum_{x_{2} \bmod \xi_{2}}^{*} e\left(\frac{d_{2} x_{2} \overline{x_{1}}}{\xi_{1}}\right) \cdots\right) \\
& =g\left(\chi^{*}, c, d_{1}\right) \sum_{x_{2} \bmod \xi_{2}}^{*} \sum_{x_{1} \bmod \xi_{1}}^{*} \chi^{*}\left(x_{1}\right) e\left(\frac{d_{2} x_{2} x_{1}}{\xi_{1}}\right)\left(\sum_{x_{3} \bmod \xi_{3}}^{*} e\left(\frac{d_{3} x_{3} \overline{x_{2}}}{\xi_{2}}\right) \cdots\right) \\
& =g\left(\chi^{*}, c, d_{1}\right) \sum_{x_{2} \bmod \xi_{2}}^{*} g\left(\chi^{*}, \xi_{1}, d_{2} x_{2}\right)\left(\sum_{x_{3} \bmod \xi_{3}}^{*} e\left(\frac{d_{3} x_{3} \overline{x_{2}}}{\xi_{2}}\right) \cdots\right) .
\end{aligned}
$$

Once again, the equalities $\left(\xi_{1}, d_{2} x_{2}\right)=\left(\left(\xi_{1}, d_{2} \xi_{2}\right), d_{2} x_{2}\right)=\left(\xi_{1},\left(d_{2} \xi_{2}, d_{2} x_{2}\right)\right)=$ $\left(\xi_{1}, d_{2}\right)$ imply that we can pull out $\overline{\chi^{*}}\left(x_{2}\right)$ from the Gauss sum. Then we have

$$
S=g\left(\chi^{*}, c, d_{1}\right) g\left(\chi^{*}, \xi_{1}, d_{2}\right) \sum_{x_{2} \bmod \xi_{2}}^{*} \overline{\chi^{*}\left(x_{2}\right)}\left(\sum_{x_{3} \bmod \xi_{3}}^{*} e\left(\frac{d_{3} x_{3} \overline{x_{2}}}{\xi_{2}}\right) \cdots\right) .
$$

The second Gauss sum $g\left(\chi^{*}, \xi_{1}, d_{2}\right)$ vanishes unless $c^{*} \mid \xi_{1} /\left(\xi_{1}, d_{2}\right)$ by Lemma 2.3. This in turn implies $c^{*}\left|\xi_{1} /\left(\xi_{1}, d_{2}\right)\right| \xi_{2}$ by (22), which is the second divisibility condition of (21). We complete the proof after repeating this process $(N-2)$ times.

Proposition 3.5. The equations (8) and (11) are equivalent.

Proof. Let $\chi$ be a Dirichlet character modulo $c$ induced from the primitive Dirichlet character $\chi^{*}$ modulo $c^{*}$. Multiply both sides of (8) by $\chi(a)$ and sum over reduced 
residue classes modulo $c$. On the left-hand side of (8), one gets

$$
\sum_{\substack{a \bmod c \\(a, c)=1}} \chi(a) L_{\boldsymbol{q}}(s, F, a / c)=\left(c / c^{*}\right)^{1-2 s} H\left(\boldsymbol{q} ; c, \chi^{*}, s\right),
$$

whereas on the right-hand side of (8), one obtains $\left(c / c^{*}\right)^{1-2 s} G\left(\boldsymbol{q} ; c, \chi^{*}, s\right)$ by making use of Lemma 3.4 and the fact that

$$
g\left(\chi^{*}, \xi_{N-2},-n\right)= \pm g\left(\chi^{*}, \xi_{N-2}, n\right),
$$

depending on whether $\chi(-1)$ is 1 or -1 . This shows that (8) implies (11).

Conversely, if we multiply both sides of (11) by $\overline{\chi(a)} / \phi(c)$ and sum over all Dirichlet characters (both primitive and nonprimitive) modulo $c$, we obtain (8) by using the orthogonality relation for Dirichlet characters. Since both of the aforementioned summations that shuttle between (8) and (11) are finite, the properties of absolute convergence and analytic continuation are preserved.

\section{Acknowledgements}

The authors thank Dorian Goldfeld and Wenzhi Luo for helpful suggestions, Matthew Young for his extensive help in organization of the manuscript and his encouragement, and Jeffrey Hoffstein, in whose class the seed for this work originated.

\section{References}

[Apostol 1976] T. M. Apostol, Introduction to analytic number theory, Springer, New York, 1976. MR Zbl

[Blomer 2012] V. Blomer, "Subconvexity for twisted L-functions on GL(3)", Amer. J. Math. 134:5 (2012), 1385-1421. MR Zbl

[Blomer et al. 2013] V. Blomer, R. Khan, and M. Young, "Distribution of mass of holomorphic cusp forms", Duke Math. J. 162:14 (2013), 2609-2644. MR Zbl

[Bump 1984] D. Bump, Automorphic forms on GL(3, $\mathbb{R})$, Lecture Notes in Mathematics 1083, Springer, Berlin, 1984. MR Zbl

[Buttcane and Khan 2015] J. Buttcane and R. Khan, " $L 4$-norms of Hecke newforms of large level", Math. Ann. 362:3-4 (2015), 699-715. MR Zbl

[Duke and Iwaniec 1990] W. Duke and H. Iwaniec, "Estimates for coefficients of $L$-functions, I", pp. 43-47 in Automorphic forms and analytic number theory (Montreal, 1989), edited by R. Murty, Univ. Montréal, 1990. MR Zbl

[Godement and Jacquet 1972] R. Godement and H. Jacquet, Zeta functions of simple algebras, Lecture Notes in Mathematics 260, Springer, Berlin, 1972. MR Zbl

[Goldfeld 2006] D. Goldfeld, Automorphic forms and L-functions for the group GL( $n, \mathbb{R})$, Cambridge Studies in Advanced Mathematics 99, Cambridge University Press, 2006. MR Zbl

[Goldfeld and Li 2006] D. Goldfeld and X. Li, "Voronoi formulas on GL(n)", Int. Math. Res. Not. 2006 (2006), art. id 86295. MR Zbl

[Goldfeld and Li 2008] D. Goldfeld and X. Li, "The Voronoi formula for GL(n, $\mathbb{R})$ ), Int. Math. Res. Not. 2008:2 (2008), art. id rnm144. MR Zbl 
[Good 1981] A. Good, "Cusp forms and eigenfunctions of the Laplacian”, Math. Ann. 255:4 (1981), 523-548. MR Zbl

[Ichino and Templier 2013] A. Ichino and N. Templier, "On the Voronou formula for GL $(n)$ ", Amer. J. Math. 135:1 (2013), 65-101. MR Zbl

[Jacquet et al. 1983] H. Jacquet, I. I. Piatetskii-Shapiro, and J. A. Shalika, "Rankin-Selberg convolutions", Amer. J. Math. 105:2 (1983), 367-464. MR Zbl

[Khan 2012] R. Khan, "Simultaneous non-vanishing of GL(3) $\times$ GL(2) and GL(2) L-functions", Math. Proc. Cambridge Philos. Soc. 152:3 (2012), 535-553. MR Zbl

[Kowalski and Ricotta 2014] E. Kowalski and G. Ricotta, "Fourier coefficients of GL $(N)$ automorphic forms in arithmetic progressions", Geom. Funct. Anal. 24:4 (2014), 1229-1297. MR Zbl

[Li 2009] X. Li, "The central value of the Rankin-Selberg L-functions", Geom. Funct. Anal. 18:5 (2009), 1660-1695. MR Zbl

[Li 2011] X. Li, "Bounds for GL(3) × GL(2) $L$-functions and GL(3) L-functions", Ann. of Math.

(2) 173:1 (2011), 301-336. MR Zbl

[Li and Young 2012] X. Li and M. P. Young, "The $L^{2}$ restriction norm of a GL 3 Maass form", Compos. Math. 148:3 (2012), 675-717. MR Zbl

[Miller 2006] S. D. Miller, "Cancellation in additively twisted sums on GL(n)", Amer. J. Math. 128:3 (2006), 699-729. MR Zbl

[Miller and Schmid 2004] S. D. Miller and W. Schmid, "Summation formulas, from Poisson and Voronoi to the present", pp. 419-440 in Noncommutative harmonic analysis, edited by P. Delorme and M. Vergne, Progress in Mathematics 220, Birkhäuser, Boston, 2004. MR Zbl

[Miller and Schmid 2006] S. D. Miller and W. Schmid, "Automorphic distributions, $L$-functions, and Voronoi summation for GL(3)", Ann. of Math. (2) 164:2 (2006), 423-488. MR Zbl

[Miller and Schmid 2011] S. D. Miller and W. Schmid, "A general Voronoi summation formula for $\operatorname{GL}(n, \mathbb{Z})$ ", pp. 173-224 in Geometry and analysis, No. 2, edited by L. Ji, Advanced Lectures in Mathematics 18, Int. Press, Somerville, MA, 2011. MR Zbl

[Miyake 1989] T. Miyake, Modular forms, Springer, Berlin, 1989. MR Zbl

[Montgomery and Vaughan 2007] H. L. Montgomery and R. C. Vaughan, Multiplicative number theory, I: Classical theory, Cambridge Studies in Advanced Mathematics 97, Cambridge University Press, 2007. MR Zbl

[Munshi 2013] R. Munshi, "Shifted convolution sums for GL(3) × GL(2)", Duke Math. J. 162:13 (2013), 2345-2362. MR Zbl

[Munshi 2015] R. Munshi, "The circle method and bounds for $L$-functions, IV: Subconvexity for twists of GL(3) L-functions", Ann. of Math. (2) 182:2 (2015), 617-672. MR Zbl

[Shimura 1975] G. Shimura, "On the holomorphy of certain Dirichlet series", Proc. London Math. Soc. (3) 31:1 (1975), 79-98. MR Zbl

[Zhou 2014] F. Zhou, "Weighted Sato-Tate vertical distribution of the Satake parameter of Maass forms on PGL $(N)$ ”, Ramanujan J. 35:3 (2014), 405-425. MR Zbl

[Zhou 2016] F. Zhou, "Voronoi summation formulae on GL(n)", J. Number Theory 162 (2016), 483-495. MR Zbl

Communicated by Philippe Michel

Received 2016-05-08 Revised 2016-07-19 Accepted 2016-09-23

ekiral@math.tamu.edu

Department of Mathematics, Texas A\&M University, College Station, TX 77843, United States

zhou.1406@math.osu.edu 


\section{Algebra \& Number Theory}

msp.org/ant

\section{EDITORS}

MANAGING EDITOR

Bjorn Poonen

Massachusetts Institute of Technology

Cambridge, USA

\author{
EDITORIAL BOARD CHAIR \\ David Eisenbud \\ University of California \\ Berkeley, USA
}

BOARD OF EDITORS

$\begin{aligned} \text { Dave Benson } & \text { University of Aberdeen, Scotland } & \text { Susan Montgomery } & \text { University of Southern California, USA } \\ \text { Richard E. Borcherds } & \text { University of California, Berkeley, USA } & \text { Shigefumi Mori } & \text { RIMS, Kyoto University, Japan } \\ \text { John H. Coates } & \text { University of Cambridge, UK } & \text { Raman Parimala } & \text { Emory University, USA } \\ \text { J-L. Colliot-Thélène } & \text { CNRS, Université Paris-Sud, France } & \text { Jonathan Pila } & \text { University of Oxford, UK } \\ \text { Brian D. Conrad } & \text { Stanford University, USA } & \text { Anand Pillay } & \text { University of Notre Dame, USA } \\ \text { Hélène Esnault } & \text { Freie Universität Berlin, Germany } & \text { Victor Reiner } & \text { University of Minnesota, USA } \\ \text { Hubert Flenner } & \text { Ruhr-Universität, Germany } & \text { Peter Sarnak } & \text { Princeton University, USA } \\ \text { Sergey Fomin } & \text { University of Michigan, USA } & \text { Joseph H. Silverman } & \text { Brown University, USA } \\ \text { Edward Frenkel } & \text { University of California, Berkeley, USA } & \text { Michael Singer } & \text { North Carolina State University, USA } \\ \text { Andrew Granville } & \text { Université de Montréal, Canada } & \text { Vasudevan Srinivas } & \text { Tata Inst. of Fund. Research, India } \\ \text { Joseph Gubeladze } & \text { San Francisco State University, USA } & \text { J. Toby Stafford } & \text { University of Michigan, USA } \\ \text { Roger Heath-Brown } & \text { Oxford University, UK } & \text { Ravi Vakil } & \text { Stanford University, USA } \\ \text { Craig Huneke } & \text { University of Virginia, USA } & \text { Michel van den Bergh } & \text { Hasselt University, Belgium } \\ \text { Kiran S. Kedlaya } & \text { Univ. of California, San Diego, USA } & \text { Marie-France Vignéras } & \text { Université Paris VII, France } \\ \text { János Kollár } & \text { Princeton University, USA } & \text { Kei-Ichi Watanabe } & \text { Nihon University, Japan } \\ \text { Yuri Manin } & \text { Northwestern University, USA } & \text { Efim Zelmanov } & \text { University of California, San Diego, USA } \\ \text { Philippe Michel } & \text { École Polytechnique Fédérale de Lausanne } & \text { Shou-Wu Zhang } & \text { Princeton University, USA }\end{aligned}$

PRODUCTION

production@msp.org

Silvio Levy, Scientific Editor

See inside back cover or msp.org/ant for submission instructions.

The subscription price for 2016 is US $\$ 290$ /year for the electronic version, and $\$ 485 /$ year (+\$55, if shipping outside the US) for print and electronic. Subscriptions, requests for back issues and changes of subscribers address should be sent to MSP.

Algebra \& Number Theory (ISSN 1944-7833 electronic, 1937-0652 printed) at Mathematical Sciences Publishers, 798 Evans Hall \#3840, c/o University of California, Berkeley, CA 94720-3840 is published continuously online. Periodical rate postage paid at Berkeley, CA 94704, and additional mailing offices.

ANT peer review and production are managed by EditFLow ${ }^{\circledR}$ from MSP.

\section{PUBLISHED BY}

- mathematical sciences publishers

nonprofit scientific publishing

http://msp.org/

() 2016 Mathematical Sciences Publishers 


\section{Algebra \& Number Theory}

Volume $10 \quad$ No. $10 \quad 2016$

Weight functions on Berkovich curves

MATTHEW BAKER and JOHANNES NICAISE

Nonvanishing of Dirichlet $L$-functions

2081

RIZWANUR KHAN and HIEU T. NGO

2093

Every integer greater than 454 is the sum of at most seven positive cubes

SAMIR SIKSEK

BERNARD LE STUM

JAN STEFFEn MÜlLER and Michael StOLL

BRUNO CHIARELLOTTO and CHRISTOPHER LAZDA

The Voronoi formula and double Dirichlet series

EREN MEHMET KiraL and FAN ZHOU

Pavel Etingof and Chelsea WALton 